\title{
Eric Schatzberg, Technology: Critical History of a \\ Concept
}

Chicago/Londres, University of Chicago Press, 2018

\section{Marcos Camolezi}

\section{(2) OpenEdition}

\section{Journals}

Édition électronique

URL : http://journals.openedition.org/artefact/7142

DOI : $10.4000 /$ artefact.7142

ISSN : 2606-9245

\section{Éditeur :}

Association Artefact. Techniques histoire et sciences humaines, Presses universitaires du Midi

\section{Édition imprimée}

Date de publication : 7 janvier 2021

Pagination : 471-475

ISBN : 978-2-8107-0706-5

ISSN : 2273-0753

\section{Référence électronique}

Marcos Camolezi, «Eric Schatzberg, Technology: Critical History of a Concept », Artefact [En ligne], 13 |

2020, mis en ligne le 23 décembre 2020, consulté le 25 décembre 2020. URL : http:// journals.openedition.org/artefact/7142 ; DOI : https://doi.org/10.4000/artefact.7142

Ce document a été généré automatiquement le 25 décembre 2020.

\section{(1) $\Theta \Theta$}

Artefact, Techniques, histoire et sciences humaines est mise à disposition selon les termes de la Licence Creative Commons Attribution - Pas d'Utilisation Commerciale - Pas de Modification 4.0 International. 


\section{Eric Schatzberg, Technology: Critical History of a Concept}

Chicago/Londres, University of Chicago Press, 2018

Marcos Camolezi

\section{RÉFÉRENCE}

Eric Schatzberg, Technology: Critical History of a Concept, Chicago/Londres, University of Chicago Press, 2018, $344 \mathrm{p}$.

1 Ce livre, défini par son auteur comme une intervention, se termine par un manifeste en faveur d'une réhabilitation de la technologie (Rehabilitating Technology). Il ne s'agit donc en aucun cas d'" un travail d'érudition neutre ", il faut plutôt y voir " une intervention dans le présent, une première étape dans la réhabilitation de la technologie en tant que concept pour l'histoire et la théorie sociale, dont l'objectif final est de façonner les technologies à des fins plus humaines" (p. 235). L'ouvrage d'Eric Schatzberg sera sans doute chaleureusement accueilli par ceux qui s'attendent à l'historicisation des concepts de «technique » et, plus particulièrement, de «technologie ». Il piquera également la curiosité de ceux qui s'intéressent à la critique historique des idées omniprésentes et pourtant faussement évidentes.

2 Faire l'histoire de "la technologie » implique de maîtriser une multiplicité de concepts dont l'éclatement empêche de les identifier facilement grâce à leur étymologie ou au caractère univoque du mot utilisé pour les nommer. Pour autant, les chapitres consacrés aux périodes ancienne, médiévale et moderne empêchent de regarder l'ouvrage comme une histoire de «la technologie » depuis son origine. Dans ce livre il n'est, par exemple, pas question de l'univers arabe, ni oriental, ou précolombien, etc. ${ }^{1}$. La tripartition aristotélicienne épistémè, technè et phronèsis, de même que la catégorie d'« arts mécaniques » créée au Moyen Âge semblent envisagées par Schatzberg comme autant de perspectives donnant à voir la place occupée par les praticiens au sein des sociétés occidentales. En fait, c'est l'émergence incessante d'un statut social et moral, 
très souvent inférieur à celui assigné au savant et au philosophe, qui est au centre de la discussion et relie les chapitres de l'ouvrage. Comme le montre Schatzberg en faisant du statut assigné à « la technologie » le fil conducteur de son propos, cette démarche permet de rapprocher des auteurs aussi différents que Bacon, Diderot et Marx. Chacun à sa manière réduit en effet la faculté d'agir (agency) des praticiens, tout en insistant sur l'importance des arts utiles et industriels.

Bien plus qu'une histoire au sens large, on peut considérer que Technology propose une histoire du statut social de «la technologie». Son objet premier consiste à observer certaines représentations de "la technologie » considérées par rapport à l'ensemble des moyens et des fins de l'être humain en société. La technologie est une activité au moyen de laquelle l'individu se réalise; pourtant, l'auteur semble vouloir rappeler qu'aucun praticien ne peut être étudié indépendamment des valeurs de la société à laquelle il appartient. Il affirme ainsi avec Leo $\mathrm{Marx}^{2}$, qu'un "vide sémantique» (semantic void) peut s'instaurer au sein d'une société et susciter la création d'un concept approprié pour désigner de nouveaux objets, de nouveaux savoirs et de nouvelles relations de production, comme ce serait arrivé avec la technology. L'auteur fait même preuve d'un certain désir républicain de découvrir dans les œuvres et controverses qui sont le miroir de l'histoire, un statut plus égalitaire accordé aux arts manuels et aux arts de l'ingénieur. Il souhaite restaurer le concept d'« art » tel qu'on l'utilisait avant le partage entre les arts utiles et les beaux-arts ; il critique l'instrumentalisme, dénonce les clivages sociaux récurrents et révèle au grand jour des écrits où « la technologie » est pensée comme une activité créatrice et normative.

4 Du point de vue de la méthode, l'une des plus grandes vertus du livre réside dans le refus de l'auteur de partir à la recherche de la définition de «la technologie». De ce fait, au lieu de proposer une investigation fondée sur la question de l'être - qu'est-ce que «la technologie»?-, l'analyse menée par Schatzberg semble motivée par la volonté de répondre à la question suivante: de quelle «technologie » parle-t-on? La deuxième qualité du volume tient au refus explicite de prendre acte d'une forme de continuité entre la technè des Anciens et la "technologie» contemporaine et de considérer que celle-ci a été inventée au début du $\mathrm{xx}^{\mathrm{e}}$ siècle aux États-Unis. Le troisième mérite de l'auteur est d'assumer sans hésitation que son livre porte sur des mots, des idées, des discours et des valeurs, plutôt que sur des objets technologiques.

De ces trois vertus découlent trois principes énoncés dans la phrase suivante: «La technologie n'est pas une entité stable» (p. 14). En premier lieu, toute recherche de la définition véritable de "la technologie » est vaine si elle ignore la relativité historique et sociale du concept. En deuxième lieu, toute analyse étymologique est suspecte, si on ne part pas du fait que "la technologie » a été elle-même l'objet de réinventions successives et simultanées au long de l'histoire. Autrement dit, le concept de «la technologie » est très loin de correspondre aux plus anciennes définitions du mot " technologie ", si bien qu'en l'espèce, toute recherche de l'origine risque de retomber, malgré elle, dans la quête de la définition véritable. En troisième lieu, l'histoire de «la technologie » doit être historicisée, c'est-à-dire que l'historien a tout intérêt à se saisir des usages locaux et temporels du concept, qui ne se communiquent universellement qu'au moyen de la ressemblance extérieure des signifiants à la racine techn- et technolog-. En bref, le livre de Schatzberg permet de constater que toute définition univoque de « la technologie » relève ou bien d'un jugement naif, ou bien d'un artifice idéologique qui l'un comme l'autre peuvent être démasqués. Le jugement naïf renvoie 
aux variations du concept de "technologie" qui compte à la fois de nombreuses traductions - parfois étranges et malheureuses - et des adaptations du terme. Celles-ci s'offrent à l'historien comme autant d'énigmes à déchiffrer. Pour ce qui est de l'artifice idéologique, il est souvent mobilisé contre les adversaires de «la technologie», face auxquels on avance des définitions supposées véritables du mot. C'est ainsi que le savant et le philosophe se sont séparés des artisans pour mieux contrôler l'espace social, contrôler des partages symboliques, manipuler l'opinion publique et partager des butins. L'attention particulière portée par Schatzberg au problème des frontières (boundaries) disciplinaires, productives et idéologiques, est à cet égard très féconde.

Dans un article publié en 2006, Eric Schatzberg avait défendu l'idée selon laquelle la technology serait née aux États-Unis dans les années 1900. C'est Thorstein Veblen qui, en traduisant le substantif allemand Technik, n'utilisa pas les cognats alors courants en langue anglaise (technics ou technique) mais eut recours au terme technology ${ }^{3}$. Dans le même article, Schatzberg montre que l'adaptation de Veblen ne consiste pas en une simple traduction des termes mais relève de l'invention du concept de technology obtenu par la réduction du champ sémantique de la Technik. En effet, parmi les deux acceptions principales de die Technik, à savoir celle concernant certains moyens capables d'amener à des fins et celle désignant l'ensemble des arts industriels, c'est la seconde qui a été prolongée par Veblen. Celui-ci choisit ainsi de définir la technology comme une Technik dans le sens que la Verein Deutscher Ingenieure (VDI) avait aidé à établir en Allemagne à partir de la seconde moitié du XIX ${ }^{e}$ siècle.

Dans son ouvrage publié en 2018, Schatzberg fait le récit de cette traduction créative dont la fortune s'est prolongée bien au-delà des années 1920. En effet, des premières définitions du concept dans les années 1900 à son établissement incontestable dans la pensée sociale anglo-saxonne dans les années 1960 et 1970, d'innombrables réappropriations ont vu le jour avant la migration de la technology jusqu'au continent européen au cours des années 1980. À cette période, "la technologie » a circulé dans des débats anglophones portant notamment sur la technocratie, le machinisme, le chômage, la science appliquée, etc.

8 S'il fallait choisir un épisode de l'histoire récente de ce concept, nous mettrions en relief l'importance prise, directement et indirectement, par les intellectuels soviétiques dans la conférence Cross Roads en 1931 (chap. 11). Schatzberg rappelle en effet que, si «les historiens des sciences anglophones ont prêté peu d'attention au rapport entre science et technologie ", " cette négligence relative a cessé brutalement avec la visite de la délégation soviétique au Congrès international de $1931 »$ (p. 187). Il souligne par exemple que Robert K. Merton, l'un des pères de la sociologie des sciences, a été profondément influencé par le paper que Boris Hessen publia en 1931 et dans lequel il soutenait que les Principia de Newton "s'ancraient dans le contexte économique, technique et idéologique du temps » (p. 190). Les tensions entre les États-Unis et l'URSS pourraient rendre étrange, aux yeux du lecteur contemporain, l'existence d'un échange théorique si fécond entre des intellectuels issus de puissances qui se sont longtemps affrontées. Toutefois, un tel échange avec les intellectuels soviétiques a dû effectivement constituer l'expérience de Merton, qui élabora quelques années plus tard la valeur de l'éthos scientifique, opposé à la possibilité d'une science nationale. On comprend également qu'après un relatif effacement de la technologie par rapport à la science au début de la Guerre froide, l'essor de «la technologie » dans les années 1950 au moment de la fondation de la SHOT - pourrait être vu comme une reprise de 
l'héritage des années 1930. Ce contexte rappelle que certains textes des années 1950 ont été valorisés à outrance, à une époque où l'histoire de "la technologie » se développait de manière certes moins fulgurante que les réflexions de Heidegger ou d'Ellul, mais avec beaucoup de solidité.

9 Le livre de Schatzberg ne peut évidemment pas être regardé comme une histoire totale de « la technologie ». S'il commence par aborder le tronc commun à toutes les cultures occidentales, il est essentiellement consacré à la branche anglo-saxonne et nordaméricaine du concept éponyme qui, ne s'est immiscé dans la culture de l'Europe continentale qu'à partir des années 1980. Pour cette raison, ce livre est également une excellente introduction à « la technologie » dont on parle, à l'échelle mondiale, depuis la fin du siècle dernier.

10 Il n'en reste pas moins vrai que des pans entiers de «la technologie » ne sont pas abordés par l'analyse de Schatzberg. C'est le cas, par exemple, de "la technique » allemande et française du $\mathrm{xx}^{\mathrm{e}}$ siècle, de celles d'Ernst Kapp mais aussi d'Alfred Espinas, Marcel Mauss et André Leroi-Gourhan. À l'instar de Schatzberg, il faut donc déterminer de quelle «technique» on parlait avant que «la technologie » ne s'immisce dans le débat public français ou dans le langage courant des organismes de financement. On saisirait certainement la raison pour laquelle des philosophes, historiens, psychologues, anthropologues, etc. parlent abondamment de « la technique » depuis les années 1930, tout comme pourquoi un certain discours technophile peut encore susciter la gêne de certains. On comprendrait du même coup pourquoi un auteur comme Michel Foucault, dont l'œuvre est tant appréciée à l'étranger, traite non pas de «technologie » mais de «technique ». Enfin, sans doute rencontrerait-on un grand nombre d'auteurs qui ont soutenu une conception très proche de celle de Schatzberg, et qui voient dans «la technologie » l'activité créatrice et normative par excellence.

11 Saluons donc l'intervention d'Eric Schatzberg, dont la lecture semble désormais indispensable pour tout historien de «la technologie » du xxe siècle et espérons que, dans un proche avenir, d'autres études viennent s'inspirer de son approche.

\section{BIBLIOGRAPHIE}

HILAIRE-PÉREZ Liliane, NÈGRE Valérie, SPICQ Delphine, VERMEIR Koen (dir.), Le Livre technique avant le $\mathrm{XX}{ }^{e}$ siècle : à l'échelle du monde, CNRS Éditions, Paris, 2017

MARX Leo, « Technology: the emergence of a hazardous concept », Social Research, 1997, 64, 3, p. 965-988, rééd. Technology and Culture, 2010, 51, 3, p. 561-577.

SCHATZBERG Eric, «Technik comes to America: changing meanings of technology before 1930 », Technology and Culture, 2006, 47, 3, p. 486-512. 
NOTES

1. Voir par exemple Hilaire-Pérez, Nègre, Spicq, Vermeir (dir.), 2017.

2. Marx, 1997 et 2010.

3. Schatzberg, 2006.

\section{AUTEURS}

MARCOS CAMOLEZI

Chercheur post-doctorant LabEx HaStec (ANR-10-LABX-85), Centre Alexandre-Koyré 